J. C. M. Richard

A. Lyazidi

E. Akoumianaki

S. Mortaza

R. L. Cordioli

J. C. Lefebvre

N. Rey

L. Piquilloud

G. F. Sferrazza-Papa

A. Mercat

L. Brochard

\title{
Potentially harmful effects of inspiratory synchronization during pressure preset ventilation
}

L. Brochard

INSERM Unit 955, Team 13, University

Paris-Est, Créteil, France

Accepted: 17 July 2013

Published online: 9 August 2013

(C) Springer-Verlag Berlin Heidelberg and ESICM 2013

J. C. M. Richard and A. Lyazidi contributed equally to this work.

Electronic supplementary material The online version of this article (doi:10.1007/s00134-013-3032-7) contains supplementary material, which is available to authorized users.

J. C. M. Richard (®) · A. Lyazidi ·

E. Akoumianaki · R. L. Cordioli ·

J. C. Lefebvre · N. Rey •

G. F. Sferrazza-Papa - L. Brochard Intensive Care Unit, University Hospital of Geneva, 4 Rue Gabrielle Perret-Gentil, 1211 Genève 14 , Switzerland e-mail: jcmb.richard@gmail.com

J. C. M. Richard · A. Lyazidi · L. Brochard School of Medicine, University of Geneva, Geneva, Switzerland

S. Mortaza - L. Piquilloud - A. Mercat Intensive Care Unit, University Hospital of Angers, Angers, France

\section{R. L. Cordioli}

Hospital Israelita Albert Einstein,

São Paulo, Brazil

J. C. M. Richard

UPRESS EA 38 30, Hôpital Universitaire

de Rouen, Rouen, France
Abstract Purpose: Pressure preset ventilation (PPV) modes with set inspiratory time can be classified according to their ability to synchronize pressure delivery with patient's inspiratory efforts (i-synchronization). Non-i-synchronized (like airway pressure release ventilation, APRV), partially i-synchronized (like biphasic airway pressure), and fully i-synchronized modes (like assistpressure control) can be distinguished. Under identical ventilatory settings across PPV modes, the degree of i-synchronization may affect tidal volume $\left(V_{\mathrm{T}}\right)$, transpulmonary pressure $\left(P_{\mathrm{TP}}\right)$, and their variability. We performed bench and clinical studies. Methods: In the bench study, all the PPV modes of five ventilators were tested with an active lung simulator. Spontaneous efforts of $-10 \mathrm{cmH}_{2} \mathrm{O}$ at rates of 20 and 30 breaths/min were simulated. Ventilator settings were high pressure $30 \mathrm{cmH}_{2} \mathrm{O}$, positive end-expiratory pressure (PEEP) $15 \mathrm{cmH}_{2} \mathrm{O}$, frequency 15 breaths/min, and inspiratory to expiratory ratios (I:E) $1: 3$ and $3: 1$. In the clinical studies, data from eight intubated patients suffering from acute respiratory distress syndrome (ARDS) and ventilated with APRV were compared to the bench tests. In four additional ARDS patients, each of the PPV modes was compared. Results: As the degree of i-synchronization among the different PPV modes increased, mean $V_{\mathrm{T}}$ and $P_{\mathrm{TP}}$ swings markedly increased while breathing variability decreased. This was consistent with clinical comparison in four ARDS patients. Observational results in eight ARDS patients show low $V_{\mathrm{T}}$ and a high variability with APRV. Conclusion: Despite identical ventilator settings, the different PPV modes lead to substantial differences in $V_{\mathrm{T}}, P_{\mathrm{TP}}$, and breathing variability in the presence spontaneous efforts. Clinicians should be aware of the possible harmful effects of i-synchronization especially when high $V_{\mathrm{T}}$ is undesirable.

Keywords Pressure preset ventilation - ARDS - APRV · BIPAP

\section{Abbreviations}

APRV Airway pressure release ventilation

ARDS Acute respiratory distress syndrome

BIPAP Biphasic positive airway pressure

$\mathrm{CV} \quad$ Coefficient of variation

I:E Inspiratory to expiratory ratio

ICU Intensive care unit

PAC Pressure assist control 


\begin{tabular}{|c|c|c|c|c|c|}
\hline PEEP & end-expiratory & $\mathrm{RR}_{\text {SPONT }}$ & $\begin{array}{l}\text { Spontaneous respiratory } \\
\text { rate }\end{array}$ & $\mathrm{VM}_{\mathrm{SPONT}}$ & $\begin{array}{l}\text { Minute ventilation } \\
\text { attributed to }\end{array}$ \\
\hline$P_{\text {MUS }}$ & Muscular pressure & SD & Standard deviation & & spontaneous breathing \\
\hline & $\begin{array}{l}\text { Pressure preset } \\
\text { ventilation }\end{array}$ & $V_{\mathrm{T}}$ & Tidal volume & $\mathrm{VM}_{\mathrm{TOT}}$ & $\begin{array}{l}\text { activity } \\
\text { Total minute ventilation }\end{array}$ \\
\hline
\end{tabular}

PS Pressure support

$P_{\mathrm{TP}} \quad$ Transpulmonary pressure

\section{Introduction}

Compelling evidence over the last decade has shown that the outcome of patients with acute respiratory distress syndrome (ARDS) can be favorably influenced by lungprotective mechanical ventilation, a strategy aiming to reduce lung strain and stress through tidal volume control $\left(V_{\mathrm{T}}\right)$ and inspiratory plateau pressure limitation [1-4]. Allowing mechanically ventilated patients with ARDS to breathe spontaneously may have physiological benefits, notably prevention of ventilator-induced diaphragmatic dysfunction. Spontaneous breathing reduces a monotonous ventilator pattern and may favor lung recruitment of the juxta-diaphragmatic lung regions, can allow reduction in sedation dosage, accelerate the weaning from mechanical ventilation, and improve hemodynamics [510]. On the other hand, spontaneous inspiratory efforts may lead to high $V_{\mathrm{T}}$, high transpulmonary pressures $\left(P_{\mathrm{TP}}\right)$, and excessive work of breathing.

Several pressure preset ventilation modes (PPV) are available on modern intensive care unit (ICU) ventilators. Differences and similarities between these modes are not always easy to identify and a great deal of confusion persists in the description of their characteristics and their effects [11]. A recent study concluded that the application of airway pressure release ventilation (APRV) requires a lot more knowledge and skills than may be apparent in the literature [12]. Using identical ventilator settings these PPV modes are indistinguishable during passive mechanical ventilation. The presence of spontaneous breathing activity can significantly change the breathing pattern, depending on the PPV mode with which the patient is being ventilated. We believe that this effect is primarily mediated by operational differences regarding the inspiratory triggering synchronization. This characteristic should be used to better classify the PPV modes of ventilation.

These bench and clinical studies were carried out to specifically assess the impact of spontaneous ventilation activity on the pattern of delivered volumes and $P_{\mathrm{TP}}$ swings during ventilation with the various PPV modes available in modern ICU ventilators. In addition to a bench test study, clinical data and ventilator recordings from two series of intubated patients suffering from ARDS were analyzed and compared to the prediction based on bench data.
Some of the results reported in the present manuscript were presented as an abstract during the 25th ESICM Annual Congress [13].

\section{Materials and methods}

Bench protocol

All available PPV modes of the following ICU ventilators were tested: Engström (General Electric, Fairfield, CT), Evita XL (Dräger, Lübeck, Germany), G5 (Hamilton Medical, Rhäzuns, Switzerland), PB 840 (Covidien, Carlsbad, CA), and Servo- $i$ (Maquet, Solna, Sweden). Their functional characteristics are described in the online supplement (Table $1 \mathrm{~S}$ ).

Based on their different levels of inspiratory synchronization (i-synchronization), the modes tested were classified into three categories: (1) Non-i-synchronized modes (e.g., APRV), in which the ventilator is never triggered by the patient, i.e., the ventilator does not attempt to synchronize the transition between the two pressure levels with patient's effort. (2) In partially i-synchronized modes [e.g., biphasic positive airway pressure (BIPAP), BiLevel, Bivent, DuoPaP, etc.] there is an i-synchronization window allowing the ventilator to be triggered if spontaneous efforts appear during this period. These modes allow the addition of pressure support (PS) for effort occurring at positive end-expiratory pressure (PEEP) level. We tested these modes with PS of $15 \mathrm{cmH}_{2} \mathrm{O}$ and without PS. (3) In fully i-synchronized modes [e.g., pressure assist control (PAC), BIPAP assist, etc.], any effort occurring at PEEP trigger the ventilator.

According to the manufacturer's recommendations, the non-i-synchronized PPV mode (APRV) in the Engström ventilator was the PAC mode with the inspiratory trigger deactivated.

Ventilator settings were similar for all modes: pressure high at $30 \mathrm{cmH}_{2} \mathrm{O}$, PEEP at $15 \mathrm{cmH}_{2} \mathrm{O}$, ventilator frequency 15 breaths/min. Two inspiratory to expiratory ratios (I:E) were tested: conventional (1:3) and inverse ratio $(3: 1)$.

A test lung, the Active Servo Lung 5000 (ASL5000, Ingmar Medical, Pittsburgh, Pennsylvania), was used to 
simulate spontaneous breathing activity. Respiratory system compliance and resistance of the simulated lung were $30 \mathrm{ml} / \mathrm{cmH}_{2} \mathrm{O}$ and $5 \mathrm{cmH}_{2} \mathrm{O} / \mathrm{l} / \mathrm{s}$, respectively, mimicking an early ARDS subject. Simulated spontaneous respiratory rates $\left(\mathrm{RR}_{\mathrm{SPONT}}\right)$ were $20 \pm 4$ and $30 \pm 4$ breaths/min (mean \pm standard deviation, SD). To mimic spontaneous variability in rate, a Gaussian distribution was used; the inspiratory time was $0.8 \mathrm{~s}$ and the muscular pressure $\left(P_{\text {MUS }}\right)$ was $-10 \mathrm{cmH}_{2} \mathrm{O}$ with a semisinusoidal inspiratory waveform profile. Following a short period without spontaneous efforts, 70 cycles were recorded at each settings combination.

\section{Data analysis}

All the 70 cycles recorded at each setting were analyzed. The resulting $V_{\mathrm{T}}$ and $P_{\mathrm{TP}}$ were computed for each cycle. Their coefficients of variation (CV), calculated as the standard deviation (SD) divided by the mean value, were assessed.

All data were stored on a laptop computer for subsequent off-line analysis using the Acknowledge software (Acqknowledge 3.7.3, Biopac Systems, Goleta, CA).

\section{Clinical studies}

\section{Observational study}

An observational study was performed in the ICU of the University Hospital of Angers. The clinical protocol was approved by the ethics committee of the hospital and the need for informed consent was waived.

\section{Methods}

Data were obtained from eight patients in the early phase of moderate to severe ARDS, ventilated in APRV mode using an Evita $\mathrm{XL}^{\circledR}$ (Dräger, Lübeck, Germany). The ventilator rate, $\mathrm{FiO}_{2}$, pressure high, PEEP, and I:E ratio were initially adjusted to maintain a mean $V_{\mathrm{T}}$ of the mandatory breaths around $6 \mathrm{ml} / \mathrm{kg}$ of ideal body weight (IBW), a pH between 7.30 and 7.45, and $\mathrm{PaO}_{2}$ between 60 and $80 \mathrm{mmHg}$ as ordered by the physician in charge. Sedation was adapted every $8 \mathrm{~h}$ to maintain Richmond Agitation Sedation Scale (RASS) score between -2 and -3 . Ventilator respiratory rate and sedation were adjusted in parallel to maintain spontaneous breathing activity $\left(\mathrm{VM}_{\mathrm{SPONT}}\right)$ displayed on the ventilator between 10 and $50 \%$ of the total minute ventilation $\left(\mathrm{VM}_{\mathrm{TOT}}\right)$ (Fig. $1 \mathrm{~S}$ ). For this purpose a specific algorithm was available at the bedside (see ESM) [14]. Over the period of ventilation with APRV, the resulting $V_{\mathrm{T}}$, the $\mathrm{VM}_{\mathrm{TOT}}$, and the $\mathrm{VM}_{\text {SPONT }}$ were continuously recorded through dedicated software (VentView, Dräger, Lübeck, Germany), which allowed last breath recording every $10 \mathrm{~s}$.

\section{Comparative study}

A comparative study was performed in the ICU of the University Hospital of Geneva. The clinical protocol was approved by the ethics committee of the hospital and informed consent was obtained from next of kin.

Four patients diagnosed with ARDS, already ventilated with one of the three PPV modes, were allocated to be successively ventilated for three 20-min periods with APRV, BIPAP, and PAC, in a random order. The ventilator settings (pressures and RR) chosen by the attending physician were used to set the ventilator with the three PPV modes tested. Sedation was kept unchanged. Airway flow was measured through a pneumotachograph (Fleish no. 2; Metabo, Epalinges, Switzerland). Airway pressure was measured using a differential pressure transducer (Validyne MP45, $\pm 80 \mathrm{cmH}_{2} \mathrm{O}$; Northridge, CA, USA). The tracings were recorded during the last $5 \mathrm{~min}$ of each period.

\section{Statistics}

Statistical analysis was performed with Statistical Package for the Social Sciences (version 16.0, SPSS, Chicago, IL, USA). All continuous variables are reported as mean $\pm \mathrm{SD}$. We used a multivariate analysis of variance to assess both the impact of the different degrees of synchronization and of the $\mathrm{RR}_{\text {SPONT }}$ on the delivered $V_{\mathrm{T}}$ and the $P_{\mathrm{TP}}$. When the overall comparisons were significant, two-by-two post hoc comparisons were performed using Tukey's test.

Clinical data were analyzed as follows. Since a different contribution of spontaneous ventilation to $\mathrm{VM}_{\mathrm{TOT}}$ was observed along the whole recording in each patient, we split the data according to the degree of spontaneous breathing activity. Six increasing levels of $\mathrm{VM}_{\mathrm{SPONT}}$ were defined, including data with $0-10,11-20,21-30,31-40$, $41-50 \%$, and having $51 \%$ or more of spontaneous breathing contributing to $\mathrm{VM}_{\mathrm{TOT}}$.

A $p$ value less than 0.05 was considered statistically significant.

\section{Results}

Bench test evaluation

During the control period without simulated respiratory effort, all PPV modes provided indistinguishable breathing patterns, whatever the ventilator, as illustrated in Fig. 2S. 
Effect of i-synchronization on mean $V_{\mathrm{T}}, P_{\mathrm{TP}}$, and variability

The presence of simulated inspiratory muscle efforts significantly altered the type of cycles recorded and their frequency (Fig. 1), thus modifying the mean delivered $V_{\mathrm{T}}$. Despite identical ventilator settings and spontaneous breathing activity, increasing the degree of i-synchronization induced more cycles with high $V_{\mathrm{T}}$ compared to nonsynchronized modes (Figs. 1, 2S). At an I:E ratio of $1: 3$ and for $\mathrm{RR}_{\text {spont }} 20$ or 30 breaths/min (Figs. 2, 3aS), the change from non- to fully i-synchronized PPV modes resulted in a marked increase in mean $V_{\mathrm{T}}(p<0.05)$ and a progressive drop in its variability. For the partially i-synchronized modes, the addition of PS further augmented the mean $V_{\mathrm{T}}$ and diminished its variability (Figs. 2, 3aS). The same effect of i-synchronization was observed on the values of $P_{\mathrm{TP}}$ and their variability (Table 1 ).

The difference between the three PPV modes was no longer statistically significant at the ratio 3:1 (Figs. 3bS, $4 \mathrm{~S})$. Compared to $1: 3$, an $\mathrm{I}: \mathrm{E}$ ratio of $3: 1$ did not change the mean $V_{\mathrm{T}}$ and its variability during non-i-synchronized PPV mode (APRV). On the contrary, the 3:1 ratio decreased the mean $V_{\mathrm{T}}$ and increased its variability during partially i-synchronized and fully i-synchronized modes.

Regardless of the operational differences existing across ventilators, the effect of i-synchronization on $V_{\mathrm{T}}$ was comparable for all ventilators tested (Table $2 \mathrm{~S}$ ).
Clinical studies

General clinical characteristics were collected for each patient in the two studies (Table 3S).

\section{Observational study}

Eight patients with ARDS were ventilated with APRV for $5 \pm 4$ days. Comparing the different periods defined by the percentage of spontaneous breathing activity contributing to $\mathrm{VM}_{\mathrm{TOT}}$, the mean $V_{\mathrm{T}}$ was $6.1 \pm 0.4 \mathrm{ml} /$ $\mathrm{kg} \mathrm{IBW}$ and remained relatively stable irrespective of this percentage (Fig. 3a). At levels of VM $\mathrm{VM}_{\mathrm{SPONT}}$ higher than $10 \%$ of $\mathrm{VM}_{\mathrm{TOT}}$, the median $\mathrm{CV}$ of $V_{\mathrm{T}}$ was higher than $30 \%$ (Fig. 3b). The observed results in patients matched the bench data reasonably well as illustrated in Fig. 3a, b.

\section{Comparative study}

The global ( $V_{\mathrm{T}}$ and its variability) and individual data $\left(V_{\mathrm{T}}\right)$ are presented on Figs. 4 and $5 \mathrm{~S}$, respectively. Increasing i-synchronization (from APRV to PAC) resulted in higher $V_{\mathrm{T}}$ and lower $V_{\mathrm{T}}$ variability (Fig. 4).
Fig. 1 Tracings of tidal volume, airway pressure, muscular pressure, and transpulmonary pressure versus time during the three PPV modes (APRV, BIPAP, and PAC). During the non-isynchronized mode four types of breaths can be observed (synchronized spontaneous and mandatory breath, spontaneous breath at high pressure or at PEEP, and mandatory breath); during the partially

i-synchronized mode, two main types of breaths are observed (synchronized spontaneous and mandatory breath, spontaneous breath at PEEP); during fully i-synchronized mode, only synchronized spontaneous and mandatory breath is observed

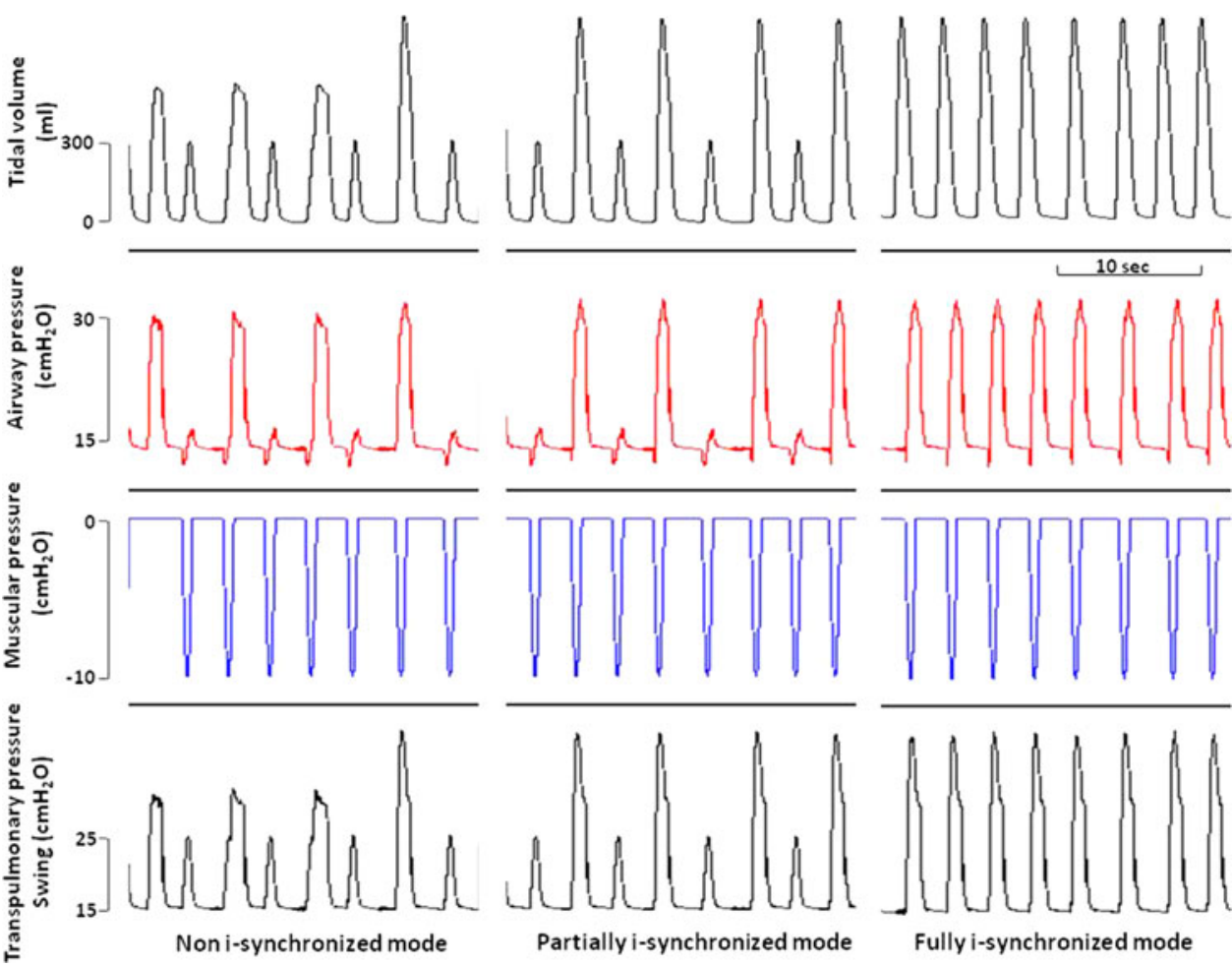




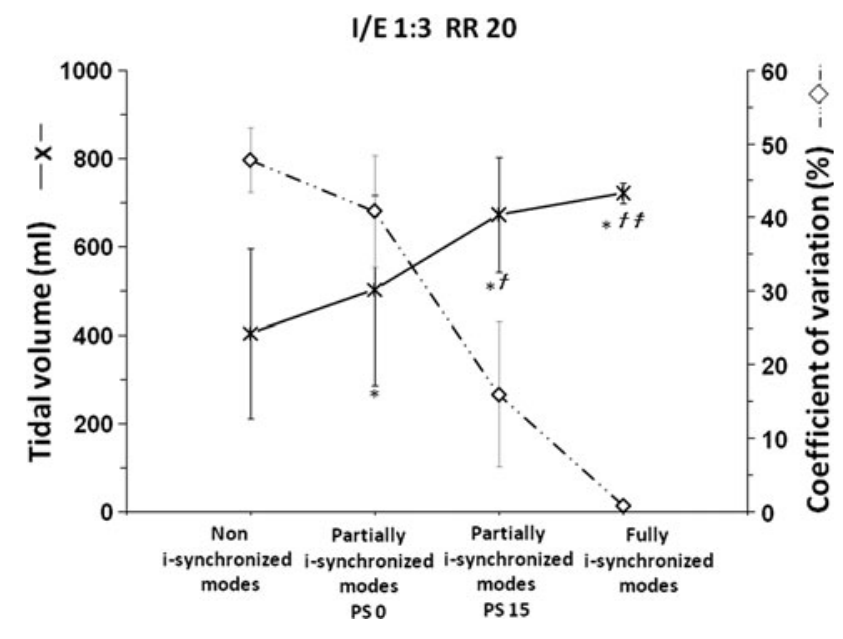

Fig. 2 The i-synchronization effect of spontaneous breathing during PPV modes. Tidal volume and its variability at a spontaneous respiratory rate of $20 \mathrm{cycles} / \mathrm{min}$ and an inspiratory to expiratory ratio of 1:3. Partially i-synchronized modes were tested with and without pressure support (PS). $* p<0.05$ vs non i-synchronized modes, ${ }^{\dagger} p<0.05$ vs partially i-synchronized modes (PS 0), ${ }^{\ddagger} p<0.05$ vs partially i-synchronized modes (PS 15)

\section{Discussion}

The bench test study shows that the presence of spontaneous breathing has different effects on the mean $V_{\mathrm{T}}$ and its variability across the various PPV modes tested and that this effect is heavily dependent on the level of i-synchronization. The lower the possibility to trigger the ventilator, the lower are the mean $V_{\mathrm{T}}$ and $P_{\mathrm{TP}}$ generated and the higher their variability. Clinical results in four ARDS patients are consistent with these observations. This suggests that there is a potential lung-protective effect of a non-i-synchronized PPV mode or, in contrast, that the full synchronization in PPV modes is associated with risks of high volumes. These findings are consistent with clinical observations in eight patients ventilated in
APRV for whom $V_{\mathrm{T}}$ remained within a safe range $(5-7 \mathrm{ml} / \mathrm{kg} \mathrm{IBW})$. These findings are useful to propose a classification of PPV modes taking into account the degree of i-synchronisation. Given the importance of tidal volume and transpulmonary pressure amplitude, clinicians must be aware of these consequences.

\section{Functional characteristics of PPV modes}

For the first time, functional differences between APRV, BIPAP, and PAC are explored and described, with potentially important clinical implications. In an attempt to better characterize the definitional criteria of BIPAP and APRV, Rose and Hawkins [11] systematically reviewed all studies performed with these modes provided that the ventilator mode characteristics were identified. The authors concluded that 'ambiguity' existed 'in the criteria that distinguish APRV and BIPAP' and that when applied with the same I:E ratio no difference existed between them. In many review articles, APRV is also referred to as an inverse ratio BIPAP suggesting that, under the same ventilator settings, APRV and BIPAP are identical $[15,16]$. Our study indicates that this similarity is only true in the absence of spontaneous breathing. In the presence of spontaneous efforts, we observed significantly higher $V_{\mathrm{T}}$ and lower $V_{\mathrm{T}}$ variability during partially and fully i-synchronized PPV (i.e., BIPAP, PAC) owing to the presence of a triggering window designed to synchronize respiratory efforts with pressure delivery. This is also due to the absence of a triggering window with non-isynchonized PPV (APRV), preventing i-synchronized cycles (with high $V_{\mathrm{T}}$ ), combined with constantly active expiratory and inspiratory valves facilitating spontaneous breathing. The high tidal volumes occasionally observed resulted from the coincidental synchronization of a timebased mandatory cycle with a patient's inspiratory effort.

Table 1 Transpulmonary pressure and its coefficient of variation at spontaneous respiratory rate $\left(\mathrm{RR}_{\mathrm{SPONT}}\right)$ of 20 and 30 cycle/min and an inspiratory to expiratory ratio of $1: 3$

\begin{tabular}{|c|c|c|c|c|c|c|c|c|}
\hline & \multicolumn{4}{|l|}{ I/E 1:3 RR 20} & \multicolumn{4}{|l|}{ I/E 1:3 RR 30} \\
\hline & $\begin{array}{l}\text { No synchronous } \\
\text { mode }\end{array}$ & $\begin{array}{l}\text { Synchronous } \\
\text { mode PS } 0\end{array}$ & $\begin{array}{l}\text { Synchronous } \\
\text { mode PS } 15\end{array}$ & PAC & $\begin{array}{l}\text { No synchronous } \\
\text { mode }\end{array}$ & $\begin{array}{l}\text { Synchronous } \\
\text { mode PS } 0\end{array}$ & $\begin{array}{l}\text { Synchronous } \\
\text { mode PS } 15\end{array}$ & PAC \\
\hline \multicolumn{9}{|c|}{ Mean value } \\
\hline Mean & 14.7 & $18.1^{\mathrm{a}}$ & $24.0^{\mathrm{a}, \mathrm{b}}$ & $25.5^{\mathrm{a}, \mathrm{b}, \mathrm{c}}$ & 14.6 & $17.0^{\mathrm{a}}$ & $24.9^{\mathrm{a}, \mathrm{b}}$ & $25.3^{\mathrm{a}, \mathrm{b}, \mathrm{c}}$ \\
\hline SD & 6.5 & 7.3 & 4.4 & 0.7 & 6.5 & 7.9 & 3.8 & 1.0 \\
\hline \multicolumn{9}{|c|}{ Coefficient of variation } \\
\hline Mean & 0.45 & 0.38 & 0.15 & 0.01 & 0.44 & 0.47 & 0.11 & 0.02 \\
\hline SD & 0.04 & 0.07 & 0.09 & 0.01 & 0.03 & 0.09 & 0.09 & 0.01 \\
\hline
\end{tabular}

Partially i-synchronized modes were tested with and without ${ }^{a} p<0.05$ vs non-i-synchronized modes pressure support (PS) added for inspiratory efforts at PEEP level ${ }^{b} p<0.05$ vs partially i-synchronized modes (PS 0 ) Values are presented as mean \pm standard deviation (SD) 

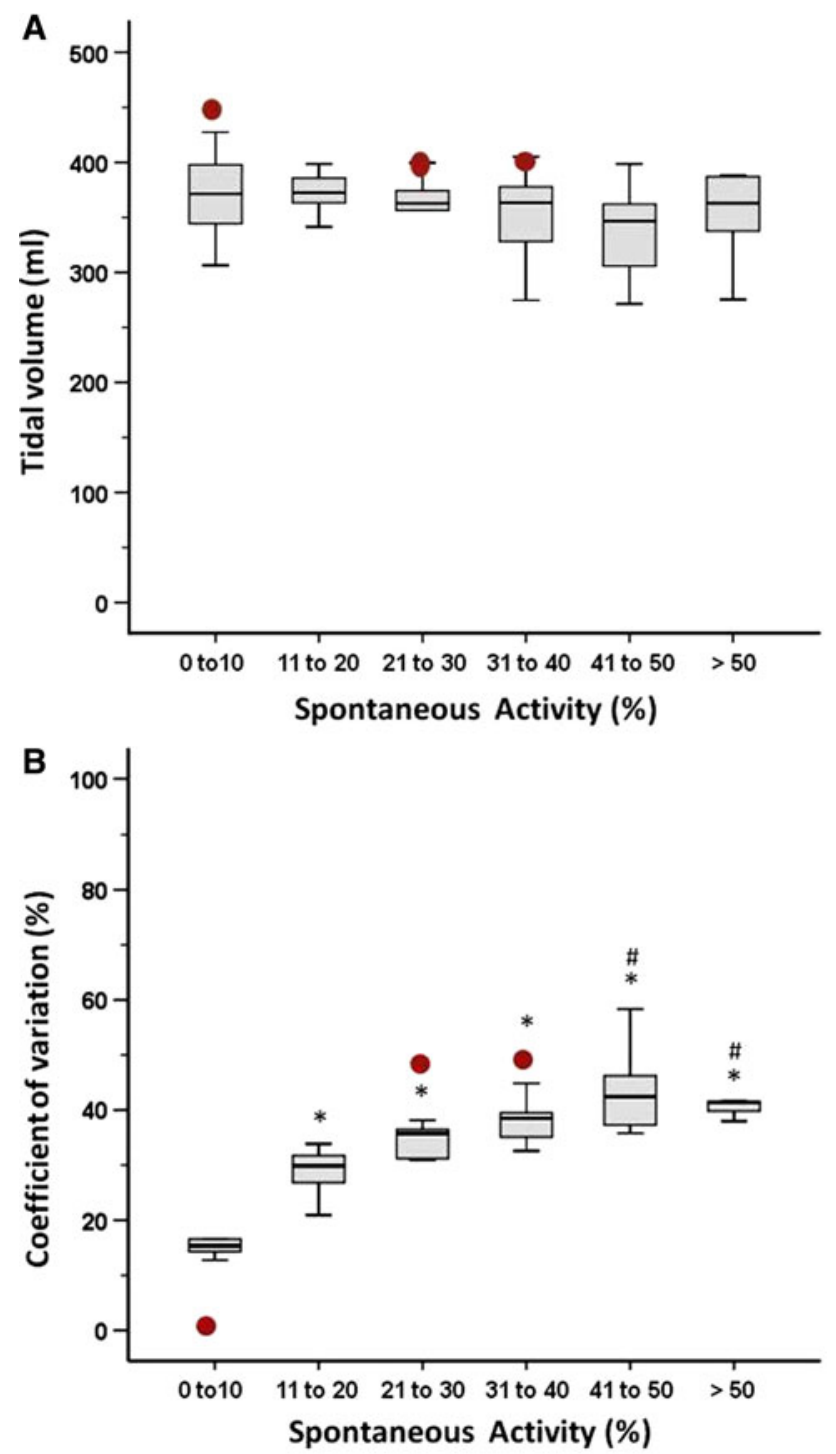

${ }^{*} p<0.05$ vs spontaneous activity of o to 10

$\# p<0.05$ vs spontaneous activity of 11 to 20

Fig. 3 a Tidal volume at different contributions of spontaneous activity to $\mathrm{VM}_{\text {TOT }}$ in patients with ARDS ventilated with APRV and during the bench study. Spontaneous activity is expressed as a percentage and calculated as the ratio of spontaneous minute ventilation to total minute ventilation. Filled red circle mean $V_{\mathrm{T}}$ during the bench study. $\mathbf{b}$ Coefficient of variation of $V_{\mathrm{T}}$ at different contributions of spontaneous activity to $\mathrm{VM}_{\mathrm{TOT}}$ in the same patients and during the bench study. Spontaneous activity is expressed as a percentage and calculated as the ratio of spontaneous minute ventilation to total minute ventilation. $* p<0.05$ vs spontaneous activity of $0-10 \%,{ }^{\#} p<0.05$ vs spontaneous activity of 11-20\%. Filled red circle coefficient of variation of $V_{\mathrm{T}}$ during the bench study

The combination of different $V_{\mathrm{T}}$ observed with APRV explains the high $V_{\mathrm{T}}$ variability (Fig. 1).

The differences observed in mean $V_{\mathrm{T}}, P_{\mathrm{TP}}$, and in $V_{\mathrm{T}}$ variability between the three PPV modes were no longer

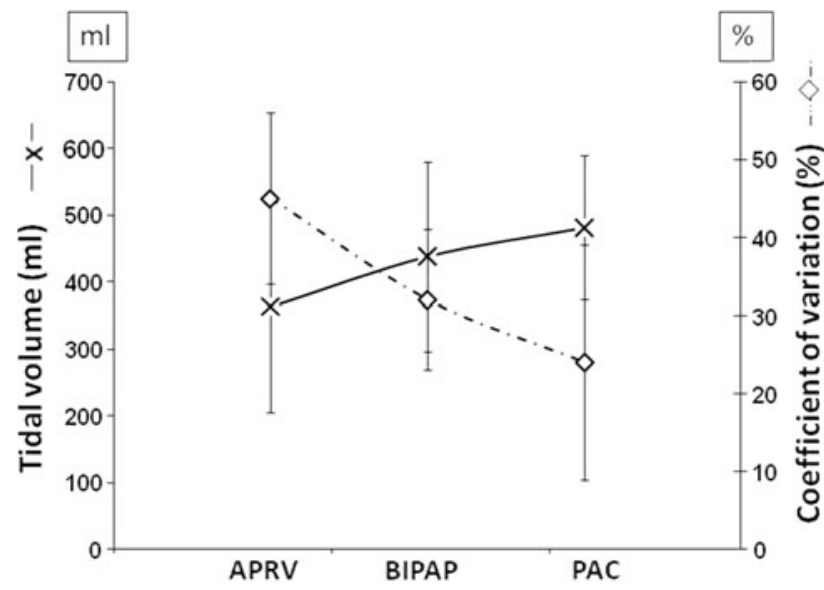

Fig. 4 Mean $V_{\mathrm{T}}$ and its variability according to the PPV modes tested during the clinical comparative study

significant at a 3:1 I:E ratio. This is explained by the fact that increasing the inspiratory time at the expense of expiratory time greatly diminishes the occurrence of i-synchronized cycles (cycles triggered by the patient at the PEEP level) which are associated with high $V_{\mathrm{T}}$. Interestingly, during APRV, $V_{\mathrm{T}}$ control and its variability were not affected by changing the I:E ratio suggesting that the breathing pattern reported with APRV results from the operational characteristics of the mode rather than from the way it is set.

In patients, the mean $V_{\mathrm{T}}$ and its variability were not altered by the different degrees of spontaneous breathing activity during APRV. This is of the utmost importance for clinicians treating patients with ARDS in which the desirable effects of spontaneous ventilation should be balanced against the hazard of high $V_{\mathrm{T}}$.

In the four patients, $V_{\mathrm{T}}$ and variability changes among modes seemed to be influenced by the relationship between patients' own respiratory rate and the set RR (ventilator). As observed in vitro (Figs. 2, 4S), the lower the ventilator expiratory time (for a given patient's RR), the lower the opportunity of synchronized breaths with BIPAP and PAC. This may explain why patient number 2 ventilated in PAC with an I:E ratio of 1:3.2 exhibited larger $V_{\mathrm{T}}$ compared to the other three patients ventilated with an I:E around 1:2. In summary, the ventilator I:E ratio and patient's RR may magnify or attenuate differences between the different PPV modes.

\section{Clinical implications}

It has been demonstrated that a ventilation strategy aiming to reduce $V_{\mathrm{T}}$ and plateau airway pressure improves the outcome of ARDS patients [1]. The concept of limiting stress (i.e., $P_{\mathrm{TP}}$ ) and strain (i.e., $V_{\mathrm{T}}$ ) has been emphasized 
in recent physiological studies [17]. Our bench study shows that in the presence of spontaneous breathing, APRV was the strategy that produced the lower $V_{\mathrm{T}}$ and $P_{\text {TP }}$ compared with the other PPV tested. Thus, a clinical benefit might be expected in ARDS patients ventilated with APRV as compared to a more conventional ventilation approach. A study by González et al. [18] failed to show any difference on clinical outcome when comparing APRV/BIPAP with volume assist-control ventilation. This study has several limitations: it was a case-matched study extracted from a large database; the level of spontaneous breathing during APRV/BIPAP was not available; no protocol was available regarding sedation target and on how to adjust ventilation during APRV. As a result, $V_{\mathrm{T}}$ was high (approximately $9 \mathrm{ml} / \mathrm{kg} \mathrm{IBW}$ ) thus limiting the conclusions of this study. A more recent prospective study [19] compared APRV with PSV with low $V_{\mathrm{T}}$ in trauma patients with acute respiratory failure. Again no difference was observed regarding outcome, but the study was underpowered and $V_{\mathrm{T}}$ and $P_{\mathrm{TP}}$ were not available.

Apart from its lung-protective role, low $V_{\mathrm{T}}$ ventilation can be associated with alveolar derecruitment leading to reduction in compliance and worsening in oxygenation [20-22]. The cyclic collapse of lung units (atelectrauma) may then promote ventilator-induced lung injury [23] that can be prevented by appropriate PEEP adjustment [24] or by the application of intermittent sighs [20-22, 25]. APRV may represent an attractive ventilation strategy allowing one to combine lung protection with low $V_{\mathrm{T}}$ and high PEEP to minimize derecruitment [15]. In contrast to the monotonous pattern observed with conventional mechanical ventilation, the high variability of $V_{\mathrm{T}}$ observed with APRV tends to mimic natural breathing. Variability has been shown to favorably influence respiratory mechanics, promote alveolar recruitment, and also improve oxygenation [3, 26, 27].

Our clinical observation in eight patients with ARDS showed that APRV ventilation, if adequately set, may preserve a low $V_{\mathrm{T}}$ (around $6 \mathrm{ml} / \mathrm{kg} \mathrm{IBW}$ ) irrespective of the contribution of spontaneous breathing activity and ensure $V_{\mathrm{T}}$ variability. To the best of our knowledge, this is the first study describing $V_{\mathrm{T}}$ and its variability during long-term application of APRV in ARDS patients. The fact that $V_{\mathrm{T}}$ was independent of the intensity of spontaneous activity suggests that APRV per se may allow one to reduce sedation without losing the control of $V_{\mathrm{T}}$. As a result of the observational character of the study we cannot attribute $V_{\mathrm{T}}$ control solely to the mode of ventilation or to the protocol design. Our clinical findings suggest that an APRV-based ventilation strategy allowing spontaneous diaphragmatic activity and protecting the lung from excessive strain is feasible.

\section{Limitations}

The bench part of this study has some limitations. Only one fixed $P_{\text {MUS }}$ and a single pattern of respiratory mechanics were tested. Furthermore, although spontaneous efforts were applied to mimic a Gaussian breathing distribution, a bench study would never be able to encompass breathing pattern complexity and interaction with the ventilator. On the other hand, the use of the lung simulator permitted one to compare the PPV modes under identical breathing conditions.

Our aim was to investigate the behavior of the three PPV modes in the presence of spontaneous breathing rather than the differences among the various ventilators. For this purpose we chose to test a series of ventilators which is not exhaustive. Nonetheless, despite slight differences in the values of $V_{\mathrm{T}}, P_{\mathrm{TP}}$, and $V_{\mathrm{T}}$ variability, the overall effect of each mode category on the parameters tested was consistent across the five ventilators tested [28, 29].

Finally, the clinical studies were limited by their small size. Nevertheless, clinical observations were highly consistent with the results of the bench study. The clinical data validated the bench test findings thus justifying the PPV mode classification proposed.

\section{Conclusion}

This study shows that, in the presence of spontaneous breathing, $V_{\mathrm{T}}$ was more efficiently controlled and variability was better preserved with non-i-synchronized pressure ventilation (APRV) independently of the ventilator settings. By contrast, i-synchronized PPV modes favored high $V_{\mathrm{T}}$ and $P_{\mathrm{TP}}$ values and reduced breathing variability. These features could be considered potentially harmful in ARDS patients. The unique features of APRV performance shown in the present study necessitate confirmation in the clinical setting.

Acknowledgments The authors are grateful to Prof. Jordi Mancebo for the critical appraisal of the manuscript. This study was supported by grants from the Société de Réanimation de Langue Française (SRLF) for Nathalie Rey and the Coordenação de Aperfeioçoamento de Pessoal de Nível Superior (CAPES)-Ministry of Education, Brazil (Bolsita da CAPES, Proc. no. BEX-18231-128) for Ricardo Cordioli.

Conflicts of interest We hereby declare that we have no conflict of interest directly related to the manuscript. LB's research laboratory received grants from several ventilator companies for specific research projects (Maquet, NAVA; Covidien, PAV; Drager, SmartCare; Philips Respironics, NIV; General Electric, FRC). 


\section{References}

1. The Acute Respiratory Distress Syndrome Network (2000) Ventilation with lower tidal volumes as compared with traditional tidal volumes for acute lung injury and the acute respiratory distress syndrome. N Engl J Med 342:1301-1308

2. Amato MB, Barbas CS, Medeiros DM, Magaldi RB et al (1998) Effect of a protective-ventilation strategy on mortality in the acute respiratory distress syndrome. N Engl J Med 338:347-354

3. Slutsky AS, Tremblay LN (1998) Multiple system organ failure. Is mechanical ventilation a contributing factor? Am J Respir Crit Care Med 157:1721-1725

4. Gattinoni L, Protti A, Caironi P, Carlesso E (2010) Ventilator-induced lung injury: the anatomical and physiological framework. Crit Care Med 38:S539-S548

5. Papazian L, Forel JM, Gacouin A, Penot-Ragon C et al (2010) Neuromuscular blockers in early acute respiratory distress syndrome. $\mathrm{N}$ Engl $\mathbf{J}$ Med 363:1107-1116

6. Putensen C, Mutz NJ, PutensenHimmer G, Zinserling J (1999) Spontaneous breathing during ventilatory support improves ventilation-perfusion distributions in patients with acute respiratory distress syndrome. Am J Respir Crit Care Med 159:1241-1248

7. Putensen C, Zech S, Wrigge H, Zinserling J, Stuber F, Von Spiegel T, Mutz N (2001) Long-term effects of spontaneous breathing during ventilatory support in patients with acute lung injury. Am J Respir Crit Care Med 164:43-49

8. Marini JJ (2012) Spontaneously regulated vs. controlled ventilation of acute lung injury/acute respiratory distress syndrome. Curr Opin Crit Care 17:24-29

9. Neumann P, Wrigge H, Zinserling J, Hinz J et al (2005) Spontaneous breathing affects the spatial ventilation and perfusion distribution during mechanical ventilatory support. Crit Care Med 33:1090-1095
10. Wrigge $H$, Zinserling J, Neumann P, Muders T, Magnusson A, Putensen C, Hedenstierna G (2005) Spontaneous breathing with airway pressure release ventilation favors ventilation in dependent lung regions and counters cyclic alveolar collapse in oleic-acidinduced lung injury: a randomized controlled computed tomography trial. Crit Care 9:R780-R789

11. Rose L, Hawkins M (2008) Airway pressure release ventilation and biphasic positive airway pressure: a systematic review of definitional criteria. Intensive Care Med 34:1766-1773

12. Sasidhar M, Chatburn RL (2012) Tidal volume variability during airway pressure release ventilation: case summary and theoretical analysis. Respir Care 57:1325-1333

13. Akoumianaki E, Rey N, Lyazidi A, Perez-Martinez N, Brochard L, Richard JCM (2012) Impact of airway pressure release ventilation (APRV) and biphasic intermittent positive airway pressure (BIPAP) modes on the lung protection in breathing lung model. Intensive Care Med 38(Suppl 1):S142

14. Sessler CN, Gosnell MS, Grap MJ, Brophy GM et al (2002) The Richmond Agitation-Sedation Scale: validity and reliability in adult intensive care unit patients. Am J Respir Crit Care Med 166:1338-1344

15. Daoud EG, Farag HL, Chatburn RL (2012) Airway pressure release ventilation: what do we know? Respir Care 57:282-292

16. Modrykamien A, Chatburn RL, Ashton RW (2011) Airway pressure release ventilation: an alternative mode of mechanical ventilation in acute respiratory distress syndrome. Cleve Clin J Med 78:101-110

17. Chiumello D, Carlesso E, Cadringher P, Caironi P et al (2008) Lung stress and strain during mechanical ventilation for acute respiratory distress syndrome. Am J Respir Crit Care Med 178:346-355

18. González M, Arroliga AC, Frutos-Vivar F, Raymondos K et al (2010) Airway pressure release ventilation versus assist-control ventilation: a comparative propensity score and international cohort study. Intensive Care Med 36:817-827
19. Maxwell RA, Green JM, Waldrop J, Dart BW et al (2010) A randomized prospective trial of airway pressure release ventilation and low tidal volume ventilation in adult trauma patients with acute respiratory failure. J Trauma 69:501-510 discussion 511

20. Richard JC, Maggiore SM, Jonson B, Mancebo J, Lemaire F, Brochard L (2001) Influence of tidal volume on alveolar recruitment. Respective role of PEEP and a recruitment maneuver. Am J Respir Crit Care Med 163:1609-1613

21. Cereda M, Foti G, Musch G, Sparacino ME, Pesenti A (1996) Positive endexpiratory pressure prevents the loss of respiratory compliance during low tidal volume ventilation in acute lung injury patients. Chest 109:480-485

22. Pelosi $\mathrm{P}$, Cadringher $\mathrm{P}$, Bottino N, Panigada M et al (1999) Sigh in acute respiratory distress syndrome. Am J Respir Crit Care Med 159:872-880

23. Muscedere JG, Mullen JB, Gan K, Slutsky AS (1994) Tidal ventilation at low airway pressures can augment lung injury. Am J Respir Crit Care Med 149:1327-1334

24. Richard JC, Brochard L, Vandelet P, Breton L et al (2003) Respective effects of end-expiratory and end-inspiratory pressures on alveolar recruitment in acute lung injury. Crit Care Med 31:89-92

25. Grasso S, Mascia L, Del Turco M, Malacarne P et al (2002) Effects of recruiting maneuvers in patients with acute respiratory distress syndrome ventilated with protective ventilatory strategy. Anesthesiology 96:795-802

26. Suki B, Alencar AM, Sujeer MK, Lutchen KR et al (1998) Life-support system benefits from noise. Nature 393:127-128

27. Ma B, Suki B, Bates JH (2011) Effects of recruitment/derecruitment dynamics on the efficacy of variable ventilation. J Appl Physiol 110:1319-1326

28. Thille AW, Lyazidi A, Richard JC, Galia F, Brochard L (2009) A bench study of intensive-care-unit ventilators: new versus old and turbine-based versus compressed gas-based ventilators. Intensive Care Med 35:1368-1376

29. Lyazidi A, Thille AW, Carteaux G, Galia F, Brochard L, Richard JC (2010) Bench test evaluation of volume delivered by modern ICU ventilators during volume-controlled ventilation. Intensive Care Med 36:2074-2080 